\title{
Una carrera contra la pérdida de la eficacia de fungicidas: 20 años de manejo de la roya asiática de la soja en Paraguay
}

\section{A race against the loss of fungicide efficacy: 20 years of managing asian soybean rust in Paraguay}

\begin{abstract}
Guillermo Andrés Enciso-Maldonado ${ }^{1 *} \odot$, Andrés Dejesús Sanabria-Velázquez ${ }^{1} \oplus$, José Arturo Schlickmann-Tank ${ }^{\mathbf{1}} \odot$, Marco Maidana-Ojeda ${ }^{1}{ }^{1}$ Centro de Desarrollo e Innovación Tecnológica. Hohenau, Itapúa, Paraguay. *Autor de correspondencia: gui77eenciso@gmail.com.
\end{abstract}

Recibido: 14 setiembre 2021

Aceptado: 6 octubre 2021

Recibido en versión modificada: 6 octubre 2021

Enciso-Maldonado, G. A., Sanabria-Velázquez, A. D., Schilickmann-Tank, J. A. \& Maidana-Ojeda, M. (2021). Una carrera contra la pérdida de la eficacia de fungicidas: 20 años de manejo de la roya asiática de la soja en Paraguay. Investigaciones y Estudios - UNA, 12 (2), 59-61. https://doi.org/10.47133/IEUNA2126b

La roya asiática de la soja (RAS), causada por el hongo Phakopsora pachyrhizi, es la enfermedad de mayor importancia en el cultivo de soja. Su primera aparición en América continental ocurrió en 2001 en Pirapó, Paraguay, y desde entonces se ha manifestado en cada temporada de producción con efectos variables (Yorinori et al., 2005). Potencialmente, este patógeno puede ocasionar la pérdida total de la producción si no se toman las medidas adecuadas.

Durante las primeras epidemias de RAS en Paraguay (2002 al 2004), el control se realizaba con fungicidas sistémicos y sitio-específicos, como los inhibidores de la desmetilación (IDM) (triazoles) y con inhibidores de la quinona externa (estrobilurinas), mientras que algunas formulaciones combinaban ambos modos de acción.
Con el paso de los años y un inadecuado manejo de la enfermedad, la eficacia de control (EC) de los fungicidas se fue reduciendo debido a que el patógeno perdió sensibilidad a los diferentes modos de acción de los ingredientes activos. Durante el 2005, salieron al mercado nuevas formulaciones que contenían mezclas de dos ingredientes activos con modos de acción distintos como la azoxystrobina + ciproconazole, la picoxistrobina + ciproconazole, la picoxistrobina + ciproconazole, la piraclostrobina + epoxiconazole o el ciproconazole + trifloxistrobina.

La aplicación de estas mezclas resultó en buenos controles de la severidad de la enfermedad y permitió obtener buenos rendimientos durante varios años. No obstante, estos fungicidas se volvieron menos eficientes en el control de la RAS hasta llegar en un punto crítico. Para obtener mejores controles, en el 2014, se introdujeron al mercado nuevas moléculas: el prothioconazole (IDM - triazolintiona), que se comercializa en mezcla con trifloxistrobina, el benzovindiflupyr y el 
fluxapyroxad, dos inhibidores de la enzima succinato deshidrogenasa (carboxamida). El benzovindiflupyr se comercializa en una formulación con azoxistrobina, mientras que el fluxapyroxad en mezcla con epoxiconazole y la piraclostrobina, siendo el primer fungicida con tres ingredientes activos en una misma formulación en comercializarse en Paraguay.

Los fungicidas evaluados en los ensayos cooperativos en Brasil mostraron reducción en el control. En 2015 se reportó que el mancozeb, un fungicida multisitio y de contacto, en combinación con fungicidas sitios-específicos permite una mayor EC en comparación con aplicaciones aisladas de los mismos, además su inclusión permitía retrasar la generación de resistencia del patógeno (Silva et al., 2015). En 2016 se incluyó el mancozeb en las aplicaciones de fungicidas para el control de la RAS en Paraguay, y en 2018 las recomendaciones incluían al mancozeb en todas las aplicaciones de fungicidas durante el ciclo productivo; hecho que elevó el costo de producción de la soja.

En Paraguay no existe actualmente una red nacional que realice pruebas de eficacia de fungicidas para el control de la RAS. La investigación que existe es realizada por el sector privado, cuyos resultados no son publicados en medios de divulgación confiables. Actualmente el costo de aplicación de fungicidas oscila entre los 120 y 145 USD ha $^{-1}, \mathrm{y}$, considerando la superficie de siembra el costo del control de la RAS puede variar entre 425 a 514 millones de dólares; solamente en la temporada normal del cultivo, es decir, excluyendo la "zafriña" o siembra alternativa de soja.

La insensibilidad del patógeno a los diferentes modos de acción no es la única causa de la disminución de la EC, ésta también varía en función de la presión de inóculo, condiciones atmosféricas, fases fenológicas, entre otras (Fleitas et al., 2015). Actualmente la decisión de aplicar fungicidas se basa en la ocurrencia de las fases fenológicas R1, R3-R5 y R5-R6. Además, a partir de la temporada 2018/2019 se introdujo el concepto de "aplicación cero", la cual consiste en una aplicación de fungicidas en la fase vegetativa (entre los 25 y 35 días después de la emergencia) para reducir el inóculo de patógenos de la soja. Las aplicaciones basadas en estas recomendaciones no garantizan una alta EC debido a que no se basa ni en la observación de síntomas ni en la presión de inóculo, sino en la experiencia de años anteriores.

Al monitorear el inóculo de $P$. pachyrhizi en el aire por medio de cazaesporas, en la temporada 2017/2018 las primeras uredospororas del patógeno se observaron en la primera semana de noviembre, en 2018/2019 en la última semana de setiembre, en 2019/2020 en la primera semana de enero, en 2020/2021 en la segunda semana de octubre y en 2021/2022 en la primera semana de octubre (datos no publicados).

Debido a que la aparición de uredosporas varía anualmente, el criterio de decisión para aplicar fungicidas basado en la ocurrencia de fases fenológicas puede generar fallas en el control o, por otro lado, aplicaciones innecesarias. A mayor presión de inóculo se reduce la EC, por lo que en una temporada como la del 2018/2019, con una aparición temprana de la RAS, las aplicaciones fueron menos efectivas para controlar la enfermedad, dado que, al momento de la primera aplicación, la soja ya presentó un nivel de severidad alto, requiriendo un mayor número de aplicaciones durante el ciclo de cultivo. 
Por el impacto económico que tiene la RAS en el país, sería beneficiosa la creación de una red nacional de monitoreo de la EC de los fungicidas disponibles en el mercado en función a la presión de inóculo en distintos puntos del país. Esto daría respuestas a las fallas de control que suelen observarse en las parcelas de los productores, además se podrían posicionar mejor los fungicidas al prevenir el desarrollo de resistencia del patógeno, creando nuevas estrategias o rotaciones de ingredientes activos, protegiendo los ingredientes activos de los fungicidas para que no pierdan su eficacia con el tiempo. Por último, la inclusión de nuevas moléculas fungicidas serán soluciones efectivas pero temporales para el control exitoso de la RAS, pero si no se realiza un manejo sustentable de los mismos, existirá una alta probabilidad de que P. pachyrhizi se vuelva insensible a la acción de éstos en poco tiempo.

Declaración de interés. Los autores declaran no tener conflicto de intereses.

\section{REFERENCIAS BIBLIOGRÁFICAS}

Fleitas, M. C., Gerard, G. S. \& Simón, M. R. (2015). Eficacia residual de fungicidas sobre la roya de la hoja del trigo y su efecto sobre componentes del rendimiento y porcentaje de proteínas en grano. Revista FAVE. Ciencias Agrarias 14 (2), 1-16. https://doi.org/10.14409/fa.v14i2.5721

Silva, L. H. C. P. D., Campos, H. D., Silva, J. R. C. \& Reis, E. M. (2015). Control of Asian soybean rust with mancozeb, a multi-site fungicide. Summa $\begin{array}{llll}\text { Phytopathologica } & 41 & \text { (1), 64-67. }\end{array}$ https://doi.org/10.1590/0100-5405/1957

Yorinori, J. T., Paiva, W. M., Frederick, R. D., Costamilan, L. M., Bertagnolli, P. F., Hartman, G. E., Godoy, C. V. \& Nunes Jr, J. (2005). Epidemics of soybean rust (Phakopsora pachyrhizi) in Brazil and Paraguay from 2001 to 2003. Plant Disease 89 (6), 675-677. https://doi.org/10.1094/PD-89$\underline{0675 .}$ 